\title{
Incidência e gravidade da retinopatia da prematuridade e sua associação com morbidade e tratamentos instituídos no Hospital Universitário Antonio Pedro, entre 2003 a 2005
}

\author{
Incidence and severity of retinopathy of prematurity and its association \\ with morbidity and treatments instituted at Hospital Antonio Pedro \\ from Universidade Federal Fluminense, between 2003 and 2005
}

\author{
Raphael de Faria Schumann ${ }^{1}$ \\ Adauto Dutra Moraes Barbosa² \\ Cristina Ortiz Valete ${ }^{3}$
}

\footnotetext{
Trabalho realizado na Faculdade de Medicina da Universidade Federal Fluminense - UFF - Niterói (RJ) Brasil.

Mestre em Medicina/Atenção Integrada à Criança e ao Adolescente. Especialista em Oftalmologia pela Universidade Federal Fluminense - UFF - Niterói (RJ) - Brasil. 2 Professor Associado de Pediatria da UFF - Niterói (RJ) Brasil. Doutor em Pediatria - Universidade Federal de São Paulo - UNIFESP - São Paulo (SP) - Brasil.

Professora Adjunta de Pediatria da UFF - Niterói (RJ) Brasil. Doutora em Saúde Coletiva, Instituto de Medicina Social da Universidade do Estado do Rio de Janeiro - UERJ - Rio de Janeiro (RJ) - Brasil.

Endereço para correspondência: Cristina Ortiz Valete. Departamento Materno Infantil. Rua Marquês do Paraná, 303 - 3ํandar - Niterói (RJ) CEP 24030-900

E-mail: cristina.ortiz@ig.com.br

Recebido para publicação em 07.06.2009

Última versão recebida em 07.01.2010

Aprovação em 14.01.2010
}

\section{RESUMO}

Objetivos: Avaliar a incidência e a gravidade da retinopatia da prematuridade em recém-nascidos pré-termo (RNPT) e sua associação com a morbidade e tratamentos instituídos, no Hospital Universitário Antonio Pedro, entre os anos de 2003 e 2005. Métodos: Estudo transversal, retrospectivo, de 73 retinopatia da prematuridade em recém-nascidos pré-termo, com idade gestatória (IG) igual ou menor que 32 semanas e/ou peso de nascimento (PN) igual ou menor que $1.500 \mathrm{~g}$, cujo exame oftalmoscópico foi realizado entre a $4^{\underline{a}}$ e a $6^{\underline{a}}$ semana de vida ou entre 32 e 36 semanas de idade gestatória corrigida. Foram registrados os seguintes dados: realização de pré-natal, tipo de parto, intercorrências na gestação e durante o parto, sexo, peso de nascimento, idade gestatória, classificação peso-idade gestacional, $\mathrm{FiO}_{2}$ máxima, tempo de uso de oxigênio, presença de persistência do canal arterial, hemorragia intracraniana, sepse, enterocolite necrosante, transfusão sanguínea no recém-nascido e uso de surfactante. O escore de gravidade utilizado foi o CRIB. Resultados: Entre os casos de retinopatia da prematuridade em recém-nascidos pré-termo estudados, 34 não apresentaram retinopatia da prematuridade $(46,6 \%)$. Dos $53,4 \%$ que apresentaram retinopatia da prematuridade, 13 tinham retinopatia da prematuridade 1 $(17,8 \%), 20$ tinham retinopatia da prematuridade $2(27,4 \%)$ e 6 tinham retinopatia da prematuridade $3(8,2 \%)$. O sexo masculino correspondeu a $45,2 \%$ da amostra. Oitenta e três por cento das mães realizaram pré-natal, $64 \%$ apresentaram alguma intercorrência na gestação e $38 \%$ durante o parto. $\mathrm{O}$ tipo de parto mais frequente foi o cesáreo (69\%). Todas as retinopatias da prematuridade em recém-nascidos pré-termo pertenciam à mesma classe de CRIB. Cerca de metade da amostra era de RN pequeno para a idade gestacional $(49,3 \%)$. A menor IG $(\mathrm{p}<0,001)$ e o menor PN ( $<<0,001)$ associaram-se à ocorrência e gravidade da retinopatia da prematuridade. Graus mais avançados de retinopatia da prematuridade associaram-se a valores médios de $\mathrm{FiO}_{2}$ mais elevados. $\mathrm{O}$ maior tempo médio de oxigenoterapia associou-se a maior ocorrência e gravidade da retinopatia da prematuridade $(\mathrm{p}<0,05)$. O Apgar de $5^{\circ}$ minuto não estava associado à ocorrência de retinopatia da prematuridade $(\mathrm{p}=0,743)$. Entre as variáveis representativas de tratamentos e morbidade, apenas a transfusão sanguínea foi incluída no modelo estudado de análise multivariada através da regressão logística hierárquica. Conclusão: Embora as características das retinopatias da prematuridade em recém-nascidos pré-termo explicassem, em parte, suas influências na gênese da doença, as transfusões sanguíneas associaramse à elevada chance de ocorrência de retinopatia da prematuridade.

Descritores: Retinopatia da prematuridade/epidemiologia; Retinopatia da prematuridade/ etiologia; Idade gestacional; Transfusão de sangue; Recém-nascido 


\section{INTRODUÇ̃̃̃O}

A sobrevida de recém-nascidos pré-termo (RNPT), cada vez menores, vem tornando frequente a ocorrência de morbidade $^{(1-2)}$, como a retinopatia da prematuridade (ROP), enfermidade fibrovascular que cursa com proliferação vascular, uma das principais causas de cegueira evitável na infância ${ }^{(3-4)}$.

A importância da ROP reside na sua frequência e no caráter de prevenção da cegueira, visto que, uma vez diagnosticada e tratada, ela dificilmente evolui para a perda total da visão ${ }^{(5)}$. Estima-se que $68 \%$ dos RNPT com menos de $1.251 \mathrm{~g}$ tenham $\mathrm{ROP}^{(6)}$ e que, na América Latina, existam cerca de 25.000 cegos em decorrência da $\mathrm{ROP}^{(4)}$. Em 2007 alguns autores concluíram que de 3.210 crianças com comprometimento visual, 7,6\% apresentavam $\mathrm{ROP}^{(7)}$.

Sabe-se que a ROP tem origem multifatorial, que ocorre na retina imatura de $\mathrm{RNPT}^{(8-10)}$ e que os fatores de risco são: a prematuridade, o menor peso de nascimento, a oxigenoterapia, a hemorragia intracraniana, a persistência do canal arterial, entre outros ${ }^{(4,11)}$. Mais recentemente, o maior número de transfusões sanguíneas também tem sido associado ao aumento da frequência dos casos de $\operatorname{ROP}^{(1,12)}$.

Considerando a gravidade da ROP, este trabalho teve como objetivo avaliar a sua frequência em RNPT e sua associação com a morbidade e tratamentos instituídos, no Hospital Universitário Antonio Pedro.

\section{MÉTODOS}

Estudo transversal retrospectivo, com coleta de dados em prontuário. Foram critérios de inclusão: Recém-nascidos com idade gestacional igual ou menor que 32 semanas e/ou peso de nascimento igual ou menor que 1.500 gramas, admitidos na UTI do Hospital Universitário Antônio Pedro (HUAP) da Universidade Federal Fluminense, entre janeiro de 2003 e maio de 2005. Os critérios de exclusão foram: recém-nascidos com malformações oculares ou síndromes genéticas com comprometimento ocular.

No período estudado, foram internados 101 RNPT e destes, 73 preencheram os critérios de inclusão.

O exame oftalmoscópico foi realizado por um único médico oftalmologista, através de oftalmoscopia binocular indireta entre a $4^{\underline{a}}$ e a $6^{\underline{a}}$ semana de vida ou entre 32 e 36 semanas de idade gestatória corrigida. Foi utilizado colírio com fenilefrina a $2 \%$ e tropicamida a $0,5 \%$, lente de $+28 \mathrm{D}$ e, quando necessário, colírio anestésico, blefarostato e indentador.

Foram registrados os seguintes dados: realização de prénatal, tipo de parto (cesáreo ou vaginal), intercorrências na gestação, intercorrências durante o parto, sexo, peso de nascimento, idade gestatória, classificação peso-idade gestatória segundo alguns autores ${ }^{(13)}$, nível de $\mathrm{FiO}_{2}$ máxima, tempo de uso de oxigênio, presença de persistência do canal arterial (PCA), hemorragia intracraniana (HIC), sepse, enterocolite necrosante (ECN), transfusão sanguínea e uso de surfactante. O escore de gravidade utilizado foi o CRIB $^{(14)}$.
Foi considerada ROP 1 a presença de linha de demarcação no mesmo plano da retina, separando a zona vascular da avascular. No estágio 2 , esta linha ganhava extensão, sendo elevada além do plano da retina, formando uma crista. A ROP 3 foi caracterizada pela presença de proliferação fibrovascular e tufos de neovasos na crista. No estágio 4, havia descolamento subtotal da retina e no estágio 5 , este descolamento era total ${ }^{(15)}$.

Os dados foram analisados com auxílio do programa Stata versão 8.0 (Stata Corporation; Texas, EUA, 2003). Os resultados foram apresentados em médias e desvios-padrão. Na análise bivariada, as diferenças entre médias foram analisadas pelo teste $t$ de Student e as diferenças de proporções pelo teste do qui-quadrado. A análise multivariada foi realizada pelo método de regressão logística hierárquica, sendo incluídas no modelo as variáveis importantes na análise bivariada (valor de $\mathrm{p}<0,10$ ) e mantidas no modelo de acordo com valor de $\mathrm{p}<0,05$. Foram construídos dois blocos de variáveis: as características dos RNPT ( $1^{\circ}$ bloco $)$ e a morbidade/ tratamento ( $2^{\circ}$ bloco).

Este estudo foi aprovado pelo Comitê de Ética em Pesquisa do Hospital Universitário Antônio Pedro (CEP CCM/HUAP 175/04).

\section{RESULTADOS}

A amostra foi constituída de 73 RNPT e destes, 34 RNPT não apresentaram ROP $(46,6 \%)$. Dos 53,4\% que apresentaram ROP, 13 tinham ROP $1(17,8 \%), 20$ tinham ROP $2(27,4 \%)$ e 6 tinham ROP 3 (8,2\%). Não foram observados ROP 4 e 5.

$\mathrm{O}$ sexo masculino correspondeu a $45,2 \%$ da amostra. Oitenta e três por cento das mães relataram ter realizado o prénatal, 64\% apresentaram alguma intercorrência na gestação e $38 \%$ apresentaram alguma intercorrência no parto. O tipo de parto mais frequente foi o cesáreo (69\%). Todos os RNPT pertenciam à mesma classe de CRIB, variando de 0 a 5 (Grau 1). Cerca de metade da amostra era de pacientes pequenos para a idade gestatória $(49,3 \%)$.

As características dos RNPT na amostra estudada encontram-se na tabela 1 .

A menor idade gestatória $(\mathrm{F}=12,196, \mathrm{p}<0,001)$ e o menor peso de nascimento $(\mathrm{F}=11,235, \mathrm{p}<0,001)$ associaram-se à ocorrência e gravidade da ROP.

Observou-se que graus mais avançados de ROP associaram-se a valores médios de $\mathrm{FiO}_{2}$ mais elevados (Tabela 2).

Tabela 1. Características dos recém-nascidos pré-termo (HUAP, 2003-2005)

\begin{tabular}{lcc|} 
Variável & Média & Desvio-padrão \\
Idade gestatória (semanas) & 30,50 & 2,4 \\
Peso de nascimento (gramas) & 1.153 & 271,5 \\
Apgar de 5 minuto & 8 & 1,9 \\
$\mathrm{FiO}_{2}$ máxima & 0,51 & 0,2 \\
$\mathrm{Tempo}^{\circ}$ de uso de oxigênio (horas) & 16,3 & 1,4 \\
\hline
\end{tabular}




\begin{tabular}{|c|c|c|}
\hline Grau de ROP & $\mathrm{FiO}_{2}$ máxima (média)* & IC $95 \%$ \\
\hline Sem ROP (n=34) & 0,37 & $0,31-0,43$ \\
\hline ROP $1(n=13)$ & 0,57 & $0,43-0,71$ \\
\hline ROP $2(n=20)$ & 0,64 & $0,51-0,78$ \\
\hline ROP $3(n=6)$ & 0,72 & $0,40-1,00$ \\
\hline
\end{tabular}

O maior tempo médio de oxigenoterapia associou-se à maior ocorrência e gravidade da $\operatorname{ROP}(\mathrm{F}=10, \mathrm{p}<0,05)$.

$\mathrm{O}$ Apgar de $5^{\circ}$ minuto não estava associado à ocorrência de $\operatorname{ROP}(\mathrm{p}=0,743)$.

Na tabela 3 destacam-se as morbidades neonatais, o sexo, tratamentos instituídos e sua associação com a ocorrência da ROP.

A análise multivariada através da regressão logística hierárquica (método "forward"), forçando a entrada da variável sexo no primeiro bloco, revelou que as características dos RNPT são responsáveis por boa parte da explicação do modelo e que, das variáveis representativas de tratamentos e morbidade, apenas transfusão sanguínea foi incluída no modelo (Tabela 4).

\section{DISCUSSÃO}

A prevalência de $53,4 \%$ de ROP encontrada no presente estudo, entre RNPT, está próxima à relatada pela literatu$\mathrm{ra}^{(11,16)}$. Esta prevalência varia de acordo com os limites impostos de idade gestatória e peso de nascimento.
A menor idade gestatória e o menor peso de nascimento são fatores classicamente associados à ocorrência de ROP. Neste estudo, estes fatores foram importantes preditores de ROP, tanto na análise bivariada quanto na multivariada. Os mesmos achados são corroborados por outros estudos ${ }^{(17-19)}$. Isto se dá pela vascularização incompleta da periferia da retina, que ocorre próximo do parto ${ }^{(20)}$.

A associação entre ROP e uso de oxigênio, observada neste estudo, de forma independente, também tem sido relatada na literatura ${ }^{(19,21-22)}$. Contudo, na análise multivariada estas variáveis não foram incluídas no modelo, possivelmente pela força das variáveis peso e idade gestatória, responsáveis por grande parte da explicação do modelo (31\%). Em outro estudo nacional, Fortes Filho e colaboradores construíram modelo de regressão logística em que conseguiram incluir somente as variáveis idade gestatória, peso de nascimento e uso de eritropoietina ${ }^{(16)}$.

\begin{tabular}{|c|c|c|c|}
\hline Variáveis & $\begin{array}{l}\text { Odds } \\
\text { ratio }\end{array}$ & p-valor & $\begin{array}{c}\mathbf{R}^{2} \\
\text { ajustado }\end{array}$ \\
\hline \multicolumn{4}{|l|}{ Primeiro bloco } \\
\hline Peso de nascimento (gramas) & 0,99 & 0,027 & \\
\hline Idade gestatória (semanas) & 0,61 & 0,006 & 0,31 \\
\hline \multicolumn{4}{|l|}{ Segundo bloco } \\
\hline $\begin{array}{l}\text { Transfusão sanguínea } \\
\text { (1=sim; 0=não) }\end{array}$ & 4,65 & 0,03 & 0,36 \\
\hline${ }^{*}=$ modelo ajustado por sexo & & & \\
\hline
\end{tabular}

\begin{tabular}{|c|c|c|c|c|c|c|c|c|}
\hline Variável & & & Sem ROP & ROP 1 & ROP 2 & ROP 3 & $\chi^{2}$ & $p$ \\
\hline \multirow[t]{2}{*}{ Sexo } & Masculino & $(45,2 \%)$ & 16 & 2 & 10 & 5 & 8,4210 & 0,0381 \\
\hline & Feminino & $(54,8 \%)$ & 18 & 11 & 10 & 1 & & \\
\hline \multirow{2}{*}{$\begin{array}{l}\text { Pequeno para a } \\
\text { idade gestatória }\end{array}$} & Não & $(50,7 \%)$ & 12 & 7 & 14 & 4 & 6,8724 & 0,0760 \\
\hline & Sim & $(49,3 \%)$ & 22 & 6 & 6 & 2 & & \\
\hline \multirow{2}{*}{ Fototerapia } & Não & $(9,6 \%)$ & 4 & 2 & 1 & 0 & 1,8110 & 0,6124 \\
\hline & Sim & $(90,4 \%)$ & 30 & 11 & 19 & 6 & & \\
\hline \multirow[t]{2}{*}{ Transfusão } & Não & $(53,4 \%)$ & 27 & 8 & 2 & 2 & 25,7020 & $<0,0010$ \\
\hline & Sim & $(46,6 \%)$ & 7 & 5 & 18 & 4 & & \\
\hline \multirow[t]{2}{*}{ Hemorragia intracraniana } & Não & $(76,7 \%)$ & 28 & 9 & 14 & 5 & 1,6650 & 0,6449 \\
\hline & Sim & $(23,3 \%)$ & 6 & 4 & 6 & 1 & & \\
\hline \multirow[t]{2}{*}{ Displasia broncopulmonar } & Não & $(38,4 \%)$ & 14 & 6 & 7 & 1 & 1,7380 & 0,6286 \\
\hline & Sim & $(61,6 \%)$ & 20 & 7 & 13 & 5 & & \\
\hline \multirow{2}{*}{$\begin{array}{l}\text { Persistência de } \\
\text { canal arterial }\end{array}$} & Não & $(76,7 \%)$ & 28 & 10 & 16 & 2 & 7,0470 & 0,0704 \\
\hline & $\operatorname{Sim}$ & $(23,3 \%)$ & 6 & 3 & 4 & 4 & & \\
\hline \multirow[t]{2}{*}{ Sepse } & Não & $(39,7 \%)$ & 15 & 6 & 7 & 1 & 2,0170 & 0,5689 \\
\hline & Sim & $(60,3 \%)$ & 19 & 7 & 13 & 5 & & \\
\hline \multirow[t]{2}{*}{ Enterocolite necrosante } & Não & $(97,3 \%)$ & 34 & 12 & 19 & 6 & 2,7070 & 0,4391 \\
\hline & Sim & $(2,7 \%)$ & 0 & 1 & 1 & 0 & & \\
\hline \multirow[t]{2}{*}{ Surfactante } & Não & $(60,3 \%)$ & 25 & 10 & 9 & 0 & 15,0520 & 0,0018 \\
\hline & Sim & $(39,7 \%)$ & 9 & 3 & 11 & 6 & & \\
\hline
\end{tabular}


Ressaltamos a associação encontrada entre transfusão sanguínea e ROP, que vem sendo sugerida pela literatura ${ }^{(23-25)}$. Esta associação, evidenciada no presente estudo, possivelmente decorre da substituição da hemoglobina fetal pela hemoglobina do tipo adulto, que por menor afinidade pelo oxigênio, libera oxigênio para os tecidos mais facilmente.

Também tem sido sugerido que a baixa atividade da ferroxidase observada no prematuro, que converte o ferro do estado ferroso para o férrico leva à existência de ferro livre, um potente formador de espécies de oxigênio altamente reagentes, capazes de causar lesão tecidual ${ }^{(26-27)}$.

\section{CONCLUSÃO}

As transfusões sanguíneas associaram-se à chance elevada de ocorrência de ROP na análise multivariada. Entretanto, vale ressaltar que pelo caráter retrospectivo do estudo, não controlamos as indicações de transfusão e os índices hematimétricos pré-transfusionais.

Assim, sugerimos a realização de estudos longitudinais, com amostra maior de RNPT, e avaliação dos fatores de risco, para estudar o real efeito destes fatores na ocorrência de ROP.

\section{ABSTRACT}

Purposes: To evaluate the incidence and the severity of retinopathy of prematurity (ROP) in preterm infants (PTI), its association with morbidity, and the treatments instituted at Antonio Pedro Hospital from the Universidade Federal Fluminense, between the years of 2003 and 2005. Methods: Transversal and retrospective study performed with 73 PTI, with gestational age (GA) of 32 weeks or less and/or birth weight (BW) of less than 1,500 g. Ophthalmoscopic exam was accomplished between 4 and 6 weeks of chronologic age or within the $32^{\text {nd }}$ to $36^{\text {th }}$ week of postconceptional age. The following data were registered: prenatal check-up, mode of delivery, gestational and childbirth complications, gender, $\mathrm{BW}, \mathrm{GA}$, weight-gestational age classification, $\mathrm{FiO}_{2}$ maximum value, oxygen use, presence of patent ductus arteriosus, intracranial hemorrhage, sepsis, necrotizing enterocolitis, blood transfusion, and surfactant use. CRIB (Clinical Risk Index for Babies) was used as severity score. Results: Among the studied newborns, 34 did not present retinopathy of prematurity $(46.6 \%)$. Out of the $53.4 \%$ that presented retinopathy of prematurity, 13 had retinopathy of prematurity $1(17.8 \%), 20$ had retinopathy of prematurity $2(27.4 \%)$ and 6 had retinopathy of prematurity $3(8.2 \%)$. Male newborns corresponded to $45.2 \%$ of the sample. $83 \%$ of the mothers accomplished prenatal care, $64 \%$ presented complications in the gestation period and 38\% during the delivery. Cesarean section was the most frequent delivery mode (69\%). All preterm infant belonged to the same CRIB score. About half of the sample was small-for-date (49.3\%). The lowest GA and BW $(p<0.001)$ were associated to the occurrence and severity of retinopathy of prematurity. The more severe cases of retinopathy of prematurity were associated to higher $\mathrm{FiO}_{2}$ average values. Longer periods of oxygen therapy use were associated with more severe retinal disease $(\mathrm{p}<0.05)$. Apgar score at the $5^{\text {th }}$ minute was not related to the occurrence of retinopathy of prematurity $(\mathrm{p}=0.743)$. Among the representative variables of treatment and morbidity, just blood transfusion was included in the studied model from multivariate analysis through hierarchical logistic regression. Conclusion: Although preterm infant features explained partly their influence on the genesis of the disease, blood transfusion was associated to a higher chance of retinopathy of prematurity.

Keywords: Retinopathy of prematurity/epidemiology; Retinopathy of prematurity/epidemiology; Gestational age; Blood transfusion; Infant, newborn

\section{REFERÊNCIAS}

1. Sinclair JC, Bracken MB. Effective care of the newborn infant. New York: Oxford University Press; 1992.

2. Schalij-Delfos NE, de Graaf ME, Treffers WF, Engel J, Cats BP. Long term follow up of premature infants: detection of strabismus, amblyopia and refractive errors. Br J Ophthalmol. 2000;84(9):963-7.

3. Phelps DL. Retinopathy of prematurity: an estimate of vision loss in the United States-1979. Pediatrics. 1981;67(6):924-5.

4. Zin A. The increasing problem of retinopathy of prematurity. J Comm Eye Health. 2001;14(1):58-9.

5. Fortes Filho JB, Eckert GU, Procianoy L, Barros CK, Procianoy RS. Retinopathy of prematurity in a tertiary center in south of Brazil. Intl J Ophthalmol. 2007;7(1):31-6.

6. Good WV, Hardy RJ, Dobson V, Palmer EA, Phelps, DL, Quintos M, Tung B; Early Treatment for Retinopathy of Prematurity Cooperative Group. The incidence and course of retinopathy of prematurity: findings for the early treatment for retinopathy of prematurity study. Pediatrics. 2005;116(1):15-23.

7. Haddad MA, Sei M, Sampaio MW, Kara-Jose N. Causes of visual impairment in children: a study of 3.210 cases. J Pediatr Ophthalmol Strabismus. 2007; 44(4):232-40

8. Ashton N, Warb B, Serpell G. Role of oxygen in the genesis of retrolental fibroplasia. A preliminary report. Br J Ophthalmol. 1953;37(9):513-20.

9. Lad EM, Nguyen TC, Morton JM, Moshfeghi DM. Retinopathy of prematurity in the United States. Br J Ophthalmol. 2008;92(3):320-5.

10. Al-Amro SA, Al-Kharfi TM, Thabit AA, Al-Mofada SM. Risk factors of acute retinopathy of prematurity. Ann Ophthalmol (Skokie). 2007;39(2):107-11.

11. Palmer EA, Flynn JT, Hardy RJ, Phelps DL, Philips CL, Schaffer DB, et al. Incidence and early course of retinopathy of prematurity. The Cryotherapy for Retinopathy of Prematurity Cooperative Group. Ophthalmology. 1991;98(11): 1628-40.

12. Bonotto LB, Moreira AT, Carvalho DS. [Prevalence of retinopathy of prematurity in premature babies examined during the period 1992-1999, Joinville (SC): evaluation of associated risks-screening]. Arq Bras Oftalmol. 2007;70(1): 55-61. Portuguese.

13. Alexander GR, Himes JH, Kaufman RB, Mor J, Kogan M. A United States national reference for fetal growth. Obstet Gynecol. 1996;87(2):163-8.

14. The CRIB (clinical risk ondex for babies) score: a tool for assessing initial neonatal risk and comparing performance of neonatal intensive care units. The International Neonatal Network. Lancet. 1993;342(8865):193-8. Erratum in: Lancet 1993;342(8871):626. Comment in: Lancet. 1993;342(8876):938. Lancet. 1993;342(8871):612-3. Lancet. 1993;342(8871):612; author reply 613. Lancet. 1993;342(8871):612; author reply 613 .

15. An international classification of retinopathy of prematurity. Prepared by an International committee. Br J Ophthalmol. 1984;68(10):690-7. 
16. Fortes Filho JB, Barros CK, da Costa MC, Procianoy RS. Results of a program for the prevention of blindness caused by retinopathy of prematurity in southern Brazil. J Pediatr (Rio J). 2007;83(3):191-3.

17. Muller H, Weiss C, Kuntz S, Akkoyun I, Schaible T. [Are there predictors for proliferative retinopathy of prematurity and is supplemental oxygen a useful conservative treatment option?] Klin Padiatr. 2005;217(2):53-60. German.

18. Yanovitch TL, Siatkowski RM, McCaffree MA, Corff KE. Retinopathy of prematurity in infants with birthweight $>1.250$ grams-incidence, severity and screening guideline cost-analysis. J AAPOS. 2006;10(2):128-34. Comment in: J AAPOS. 2007;11(2):208-10; author reply 210.

19. Dutta S, Narang S, Narang A, Dogra M, Gupta A. Risk factors of threshold retinopathy of prematurity. Indian Pediatr. 2004;41(7):665-71.

20. Weakley DR Jr, Spencer R. Current concepts in retinopathy of prematurity. Early Hum Dev. 1992;30(2):121-38.

21. Graziano RM. Retinopatia da prematuridade: contribuição ao estudo da ocorrência e análise dos fatores de risco [tese]. São Paulo: Universidade de São Paulo; 1994.
22. Sola A, Chow L, Rogido M. [Retinopathy of prematurity and oxygen therapy: a changing relationship]. An Pediatr (Barc). 2005;62(1):48-63. Spanish.

23. Hesse L, Eberl W, Schlaud M, Poets CF. Blood transfusion. Iron load and retinopathy of prematurity. Eur J Pediatr. 1997;156(6):465-70.

24. Hirano K, Morinobu T, Kim H, Hiroi M, Ban R, Ogawa S, et al. Blood transfusion increases radical promoting non-transferrin bound iron in preterm infants. Arch Dis Child Fetal Neonatal Ed. 2001;84(3):F188-93.

25. Dani C, Reali MF, Bertini G, Martelli E, Pezzati M, Rubaltelli FF. The role of blood transfusions and iron intake on retinopathy of prematurity. Early Hum Dev. 2001; 62(1):57-63.

26. Evans PJ, Evans R, Kovar IZ, Holton AF, Halliwell B. Bleomycin-detectable iron in the plasma of premature and full-term neonates. FEBS Lett. 1992;303(2-3): 210-2

27. Dani C, Martelli E, Bertini G, Pezzati M, Rossetti M, Buonocore G, et al Effect of blood transfusions on oxidative stress in preterm infants. Arch Dis Child Fetal Neonatal Ed. 2004;89(5):F408-11.

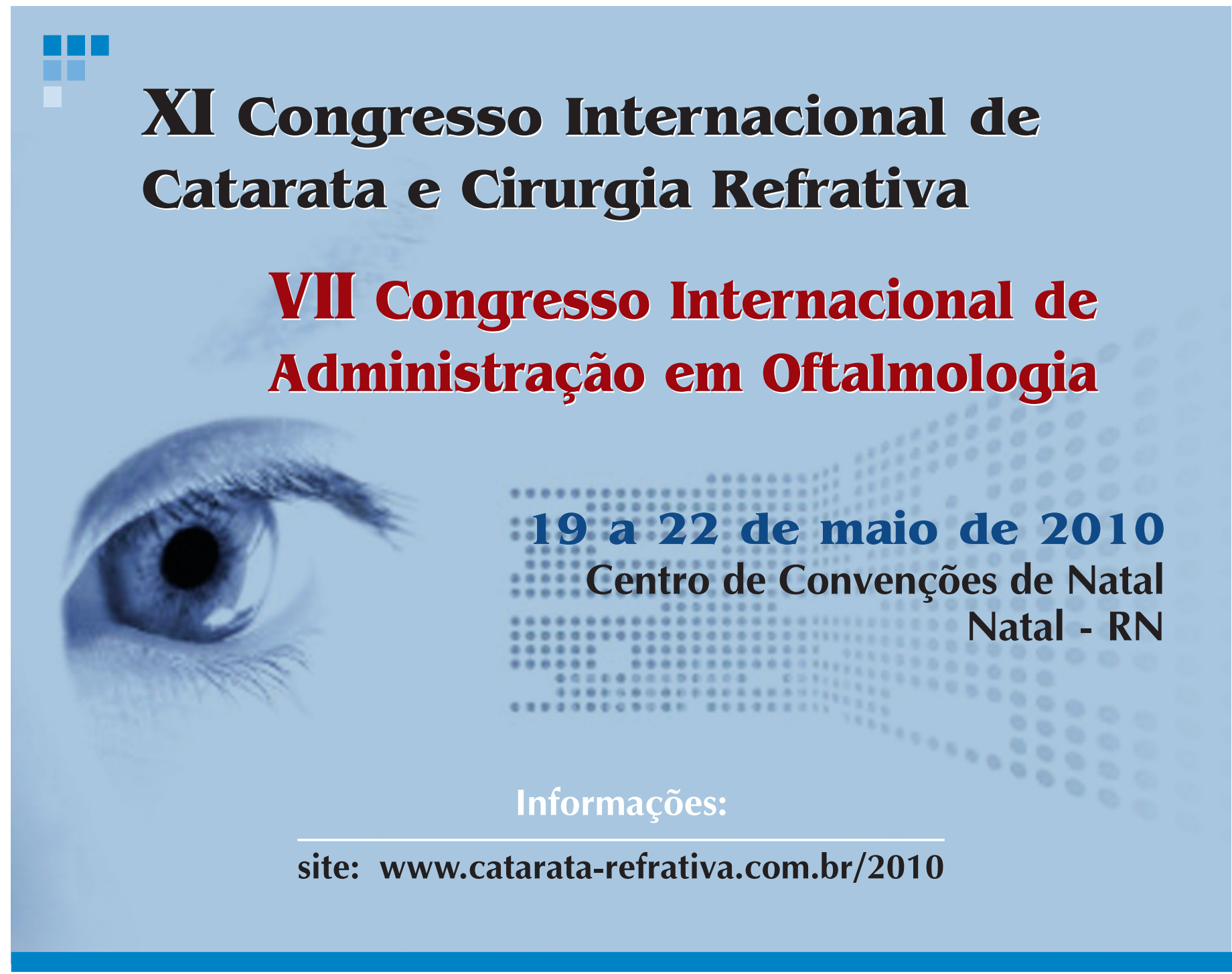

